East African Medical Journal Vol. 80 No. 12 December 2003

MORBIDITY AND MORTALITY OF NEONATES ADMITTED IN GENERAL PAEDIATRIC WARDS AT KENYATTA NATIONAL HOSPITAL

D. E. Simiyu, MBChB, MMed, Cert Neonatology (Tel Aviv), Lecturer, Department of Paediatrics and Child Health, College of Health Sciences, University of Nairobi, P.O. Box 19676, Nairobi, Kenya

Request for reprints to: Dr. D. E. Simiyu, Department of Paediatrics and Child Health, College of Health Sciences, University of Nairobi P.O. Box 19676, Nairobi, Kenya

\title{
MORBIDITY AND MORTALITY OF NEONATES ADMITTED IN GENERAL PAEDIATRIC WARDS AT KENYATTA NATIONAL HOSPITAL
}

\author{
D. E. SIMIYU
}

\begin{abstract}
Background: Neonatal morbidity and mortality is high in developing countries. Facilities for specific neonatal care are limited in Kenya with no neonatal intensive care unit in the public health institutions. The new born unit at Kenyatta National Hospital (KNH) cannot accommodate all neonates so a number of these are admitted to the general paediatric wards.

Objectives: To establish the morbidity and mortality of neonates admitted to the general wards at KNH and to review the medical interventions applied in the management and make recommendations that will improve on this.

Design: A retrospective study.

Setting: Kenyatta National Hospital general paediatric wards-Nairobi, Kenya.

Subjects: All babies who were admitted to the general paediatric wards at KNH from January to December 2000.

Results: A total of 308 records of neonates admitted to the general paediatric wards at $\mathrm{KNH}$ in the year of study were found and retrieved. The mortality was $315 / 1000$ neonates admitted. Of the 308 admissions, $33.4 \%$ were low birth weight (LBW), $5 \%$ being very low birth weight (VLBW). The common diagnoses at admission or discharge were suspected sepsis $71 \%$ (confirmed sepsis $8.4 \%$ ), jaundice 35\%, pneumonia 32\%, omphalitis $28 \%$, dehydration $14 \%$, apnoec attacks $13 \%$, and hypothermia $6 \%$. Eighty nine $(29 \%)$ of the infants were not investigated at all. The mean duration of stay was nine days for those who lived to discharge and four days for those who died. Of those who died, $49 \%$ died within 24 hours of admission. The frequently cited cause of death was suspected sepsis, pneumonia, dehydration and hypothermia. There were only six admissions to the general purpose intensive care unit referred from the wards. The independent predictors of mortality were low birth weight, apnoec attacks, hypothermia and dehydration $(\mathbf{p}<0.05)$.

Conclusion: The mortality rate for neonates admitted to the general paediatric wards is high with almost half the deaths occurring in the first twenty four hours of admission. While the wards are doing commendable work, they need support by improving laboratory back up for improved diagnostic ability and to improve supportive care such as maintenance of fluid electrolyte balance, temperature regulation and access to intensive care facilities.
\end{abstract}

\section{INTRODUCTION}

Neonates are admitted to the general paediatric wards of KNH when they don't meet the criteria of admission to the new born unit. Several studies have been done in the new born unit to determine the morbidity and mortality of new borns in the unit but none have been done for the general wards (1-3).This study therefore has no precedent to which comparisons can be made except for those studies from the new born unit.

The general wards do not have specialised staff in neonatology and are usually equipped with very basic tools such as some incubators and a phototherapy box. There being no neonatal intensive care unit in the hospital, these children compete with those from the new born unit and other hospital departments for admission to the general intensive care unit when the need arises.

From past mortality records, it appears that mortality rate for neonates in the general wards may be higher than in the new born unit(4). In the new born unit, major causes of morbidity and mortality were found to be immaturity, respiratory distress, infection and perinatal asphyxia(3).

The infants admitted to the general wards differ from those admitted in the new born unit since they have come from home. They may have previously been 
discharged from a health institution after delivery or may have been born at home. They are also older at the time of admission. The causes of death among this infants have not been studied. Due to the mentioned differences, these causes may be different from those found in the new born unit.

With this background, this study aims to document the morbidity and mortality of these infants and review their management to identify areas that need improvement to optimize their care.

\section{MATERIALS AND METHODS}

The study was conducted at the Kenyatta National Hospital. The records of all neonates admitted to the general wards from January to December 2000 were retrieved from the records department. The neonatal period was defined as that time from birth to twenty eight days of age.

A pre-coded data sheet was used to extract information from each record identifying the patient by hospital inpatient number and study number given in consecutive sequence. The data extracted included maternal age, parity, marital status, place of delivery, and mode of delivery. For the infant the data extracted included birth weight, weight at admission, discharge or death, age at admission, sex, gestational age, diagnoses at admission and on discharge or death, duration of stay, investigations done, and management events such as antibiotic use (type and duration), blood transfusions, exchange transfusion, phototherapy, surgery, oral feeds (type and method of feeding) intravenous fluids, (type and age at start of infusion and duration).

Ethical Considerations: This being a retrospective study, ethical considerations were fulfilled by obtaining permission from the ethics and research committee of the Hospital. Thereafter inpatient registration number(5), were used to identify the participants and not names hence ensuring confidentiality.

Data management: The data was entered in a computer and analysed on the SPSS programme. Percentages, ratios, and correlations were derived where appropriate and finally logistic regression was done to identify the predictors of mortality.

\section{RESULTS}

Three hundred and eight records were retrieved. Some fourty records lacked information on maternal characteristics such as on gestational period, marital status and Apgar score.

Maternal attributes: Maternal age was reported for only $44(15 \%)$ and of these 12 were teenage mothers. Marital status was recorded for $277(90 \%)$ of the mothers with majority $222(72 \%)$ being married. There was no significant difference in the mortalities between neonates of married mothers and those of single mothers.

Maternal parity information was available for 272 $(88 \%)$. It ranged from parity one to nine. The majority were first time mothers $138(45 \%)$. One hundred and ninety two $(62 \%)$ of the mothers had attended at least two antenatal visits prior to delivery. There was no information on antenatal attendance or lack of it for $51(17 \%)$ of the mothers. The rest $(21 \%)$ had not attended antenatal clinic.

Majority of the mothers $191(62 \%)$ delivered in a health facility. This included KNH 21(7\%) and Pumwani maternity hospital $47(15 \%)$, born on the way to hospital $11(4 \%)$, the remaining $105(34 \%)$ were born at home (Figure 1)

\section{Figure 1}

Place of birth

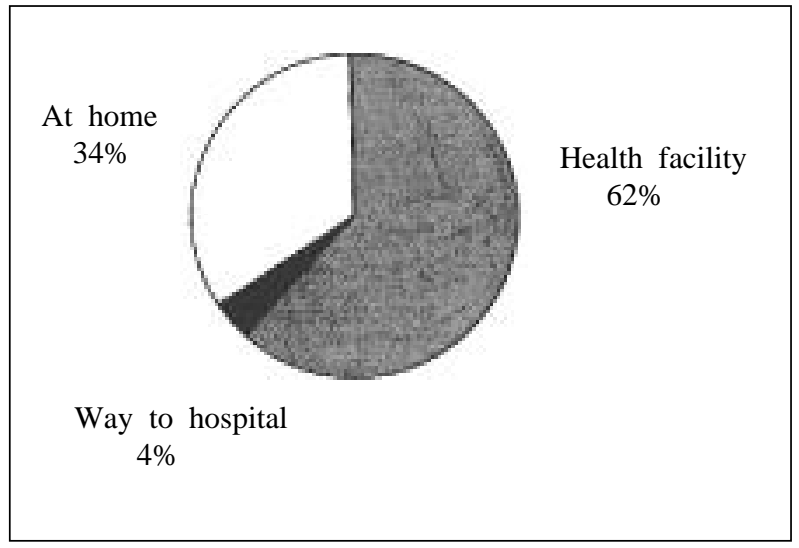

Mode of delivery was not specified for $5 \%$ while only $4 \%$ were born by Caesarian section. The rest $(91 \%)$ were born vaginally with three breech and two vacuum extractions. In this study, survival was not influenced by maternal factors such as parity, antenatal attendance nor mode of delivery.

Infants attributes: The mean age at admission was 9.6 days with a range of 4 to 25 days. Of the 308 neonates; 180 were males and 124 were females giving a male to female ratio of $1.5: 1$. Ninety seven $(31.5 \%)$ of the infants died making a mortality rate of 315/1000. In total $91(33.4 \%$ ) had low body weight at admission. Just under half, $46(47 \%)$ of those with low body weight died. Low body weight contributed significantly to death. Weight at admission was the one used rather than birth weight as many infants were born at home and came to hospital several days later. On admission, $27(18.51 \%)$ were not weighed (Table 1).

\section{Table 1}

Admission weight and mortality

\begin{tabular}{llclc}
\hline Admission weight $(\mathrm{g})$ & Alive & Dead & Total & Mortality(\%) \\
\hline$\leq-1000$ & 0 & 2 & 2 & 100 \\
$1001-1500$ & 8 & 11 & 19 & 57.8 \\
$1501-2000$ & 15 & 24 & 39 & 61.5 \\
$2001-2500$ & 25 & 18 & 43 & 41.8 \\
$2501-3000$ & 50 & 17 & 67 & 25.4 \\
$\geq 3000$ & 91 & 20 & 111 & 18.0 \\
Not weighed & 22 & 5 & 27 & 18.5 \\
\hline Total & 211 & 97 & 308 & 31.5 \\
\hline
\end{tabular}


Among those infants with body weight $\leq 2500 \mathrm{~g}$, mortality was $53.5 \%$ while among those $\geq 2501 \mathrm{~g}$ mortality was $21 \%$. So low body weight of $2500 \mathrm{~g}$ and less carried a higher mortality. The average duration of stay was nine days with a range of 16 hours to 102 days for those who lived to discharge. For those who died, the average duration of stay was four days with a range of 1 hour to 23 days.

Majority of the deaths occurred in the first week of admission(83.5\%) especially in the first 24 hours $48(49 \%)$. The causes of mortality at this early period were apnoec attacks 30(31\%), pneumonia 40(41\%) and hypothermia $4(4 \%)$. Most infants had more than one diagnoses at admission or during their stay in the wards. The most frequent diagnoses were suspected sepsis, jaundice, pneumonia, omphalitis and low body weight (Table 2).

Table 2

Frequency of diagnoses and mortality

\begin{tabular}{llll}
\hline Diagnosis & Total & Dead & Mortality $(\%)$ \\
\hline & & & \\
Suspected sepsis & 219 & 77 & 35 \\
Jaundice & 106 & 24 & 23 \\
Pneumonia & 99 & 48 & 48 \\
Omphalitis & 85 & 19 & 22 \\
Low Body Weight & 79 & 46 & 58 \\
Dehydration & 42 & 23 & 55 \\
Apnoeic attacks & 39 & 32 & 82 \\
Anaemia & 37 & 8 & 22 \\
Asphyxia & 34 & 10 & 29 \\
Confirmed Sepsis & 26 & 4 & 15 \\
Convulsions & 24 & 14 & 58 \\
Conjuctivitis/Ophthalmia & 21 & - & - \\
Diarrhoea & 21 & 9 & 43 \\
Hypothermia & 19 & 14 & 74 \\
Congenital Anomalies & 16 & 6 & 38 \\
Tetanus & 12 & 11 & 92 \\
Hypoglycaemia & 10 & 7 & 70 \\
Others & 28 & 9 & 32 \\
\hline
\end{tabular}

Infection: The most frequent diagnoses was probable sepsis $219(71 \%)$ with a mortality of $35 \%$. A total of $43(19 \%)$ babies with probable sepsis had a blood culture report. Twenty six had a positive blood culture (confirmed sepsis) making a rate of $8.4 \%$ (one culture had two organisms isolated). Meningitis was clinically suspected in 10 patients of whom seven had CSF cultures taken which were all negative. Of the 11 neonates diagnosed with tetanus only one survived to discharge. All of those with tetanus were born at home and at term. While there were 14 cases of documented prolonged rupture of membranes $>24$ hours, only one of these had confirmed sepsis on blood culture.

The common bacterial isolates were coagulase negative staphylococci and citrobacter. In this study, gram positive organisms were responsible for half of the septicaemia (Table 3)
Table 3

Range of isolates

\begin{tabular}{ll}
\hline Bacteria & Number \\
\hline Gram positive & \\
$\quad$ Coag-ve staph & 8 \\
$\quad$ Staphaureus & 5 \\
$\quad$ Enterococci & 1 \\
Total & $14(51.8 \%)$ \\
Gram negative & \\
$\quad$ Citrobacter & 6 \\
$\quad$ Klebsiella & 3 \\
Others & 4 \\
Total & $13(48.1 \%)$ \\
\hline Total & 27 \\
\hline
\end{tabular}

The others were alkaligenes fecalis, acinetobacter, pseudomonas aeruginosa and escherichia coli with one report each. One culture had mixed growth of coagulase negative staphylococci and enterococci. Other recorded infections were two cases of vericella and five of malaria. The infants with confirmed sepsis tended to stay longer in the wards with an average stay of 16.6 days as compared to the overall average duration of nine days. Haematological: A total of $106(34 \%)$ had diagnosis of jaundice made with mortality of 24(23\%). Blood grouping and Coombs test for mother and baby were done in only ten pairs. Otherwise 192 bilirubin estimates where done with some babies having upto four estimates done. One hundred and two (33\%) infants underwent phototherapy. The average duration of phototherapy was five days with a range of four hours to 19 days. Eleven (11\%) infants underwent exchange transfusion making a rate of 1 in 9 with jaundice undergoing exchange transfusion. One mother of the infants undergoing exchange transfusion died at delivery hence her blood group and Coombs test to make a pair with her infant was not done. Thirty seven $(12 \%)$ were diagnosed with anaemia both clinically and by laboratory haemoglobin estimate, nine (24\%) were subsequently transfused. The reasons for not transfusing the others were given in eight cases as lack of blood from the blood bank. For the remaining 20 no reasons were given for withholding transfusion. Eight (22\%) of the infants with anaemia who were not transfused died.

\section{SUPPORTIVE CARE}

Intravenous fluids: One hundred infants (32\%) were started on intravenous fluids. From the records it was not possible to ascertain at what time after admission that the fluids were started. However of those on intravenous fluids 54\% were given for less than 24 hours. Types of fluids administered included $10 \%$ dextrose, $10 \%$ dextrose with added electrolytes in the form of normal saline and $15 \%$ potassium chloride, $5 \%$ dextrose and half strength darrows. Sixty one percent of the babies on intravenous fluids died. 
Oral feeding: Majority 68 (70\%) of infants who died were not tolerating enteric feeding either by cup, nasogastric tube or breast feeding. There had been no attempt to feed 19(20\%) of these due to poor state. For the others, breast milk was used in 228(74\%) infants, formula in ten infants and cows milk in two infants.
Reasons for none use of breast milk included maternal death (one), being abandoned (seven), mother unwell and admitted elsewhere (two) and HIV disease (two). Incubator care: No records were kept for those (if any) who had incubator care.

Table 4

Number of infants investigated/ type of investigation

\begin{tabular}{lll}
\hline & No. & $\%$ \\
\hline None & 89 & 29 \\
Bilirubin estimate & 102 & 33 \\
Haemogram & 109 & 35 \\
Blood group infant & 29 & 9.4 \\
Blood group mother/infant & 10 & 3.2 \\
Blood culture & 43 & 14 \\
Urea/electolytes & 43 & 14 \\
Blood slide for malaria & 42 & 13.6 \\
Blood sugar & 18 & 5.8 \\
Others & 30 & 9.7 \\
\hline
\end{tabular}

Table 5

Independent predictors of mortality

\begin{tabular}{|c|c|c|c|c|c|c|}
\hline Variable & $\begin{array}{l}\text { Mortali } \\
\text { Dead }\end{array}$ & $\begin{array}{l}\text { each ce } \\
\text { Total }\end{array}$ & $\%$ & Odds ratio & $\begin{array}{l}95 \% \\
\text { confidence } \\
\text { Interval }\end{array}$ & $\begin{array}{l}\text { Statistical } \\
\text { significance }\end{array}$ \\
\hline Low Body Weight & 46 & 79 & 57 & 0.17 & $0.09-0.33$ & $\mathrm{P}=0.000002$ \\
\hline No Investigations & 55 & 89 & 57 & 5.27 & $3.02-9.23$ & $\mathrm{P}=0.00025$ \\
\hline Apnoec attacks & 32 & 39 & 82 & 0.07 & $0.03-0.18$ & $\mathrm{P}=0.000025$ \\
\hline Hypothermia & 14 & 19 & 74 & 0.14 & $0.04-0.45$ & $\mathrm{P}=0.00004$ \\
\hline Pneumonia & 48 & 99 & 48 & 2.90 & $1.70-4.94$ & $\mathrm{P}=0.0004$ \\
\hline Dehydration & 23 & 42 & 55 & 0.32 & $0.16-0.65$ & $\mathrm{P}=0.0004$ \\
\hline Sepsis* & 81 & 219 & 32 & 0.34 & $0.09-1.08$ & $\mathrm{P}=0.04$ \\
\hline
\end{tabular}

Investigations: Eighty nine(29\%) infants were not investigated at all. One hundred and nine $(35 \%)$ had a haemogram performed but only $70(64 \%)$ results were received back from the laboratory. The next common investigation was bilirubin estimation in 102(33\%) of the neonates. Those who underwent exchange transfusion had serial bilirubin estimates done. The next common investigations were urea, electrolytes and blood culture with 43(14\%) each. Many babies had more than one investigation done hence the totals in column 1 are not equal to 308 (Table 4).

Specific care: Only six neonates were admitted to the ICU for ventilator support although 39 had significant respiratory disease to require ventilation.

Antibiotics: There was liberal use of antibiotics such that except for eight (2.6\%) all neonates admitted were started on parenteral antibiotics even for the 89 whose diagnosis was neither probable nor confirmed sepsis. The most common empirical combination was that of crystalline penicillin and gentamicin . This combination was started in practically all the neonates. This was changed to a second line of a cephalosporin and gentamicin or amikacin in 37 infants. This change was dictated by culture results in $13(35 \%)$. The rest of the culture reports were received long after the patients had been discharged or died. Four infants were started on second line immediately on admission based on clinical presentation. The cephalosporins commonly used were ceftriaxone, ceftazidine and cefuroxime in that order. Excluding the 48 neonates who died within 24 hours of admission, the average duration of parenteral antibiotic therapy was seven days.

Correlations: To determine significant predictors of mortality in infants admitted to the general wards, multivarient analysis by step-wise logistic regression was performed with mortality as the dependant variable. (Table 5).

The strongest predictor of mortality in neonates admitted to the general wards was low body weight followed by lack of investigations, apnoec attacks, hypothermia, pneumonia, dehydration and sepsis 
(confirmed or probable) in that order. The variables that were not independently associated with mortality were maternal parity, marital status, attendance at antenatal clinic, place of delivery and mode of delivery. For the infants, sex, jaundice and omphalitis were not independently associated with mortality.

\section{DISCUSSION}

Being a retrospective study, it suffered from the drawback of the researcher not being in control of the processes. In this particular case, poor record keeping was a major drawback. The study is unique in the sense that there is no previous similar study locally hence comparisons are difficult to make. The nearest comparisons are with studies related to care of newborns in specially designated areas i.e newborn units. The differences that arise between infants in a new born unit and those in a general ward stem from;

(i) lack of personel specialised in the care of newborns in the general wards

(ii) lack of specialised equipment in the general wards(1).

Maternal attributes: In this study, maternal attributes such as marital status, parity, antenatal clinic attendance, mode or place of delivery had no influence on the mortality. Others have also shown this to be true (3,68 ). The reason why these maternal variables had no influence in this study could be that the neonates admitted to the general wards had attributes that negated the influence of these variables. The average age at admission was high (9.6 days) hence the infants may have overcome or survived the influence of maternal variables.

Infant attributes: The overall mortality rate of 315/1000 admissions though high is similar to that reported in the new born unit of the same hospital and elsewhere in developing countries. The contribution of low body weight to mortality in this study was high. This has been shown by various researchers to hold true both locally and elsewhere $(2,3,6,9,10)$. This is a category of infants who require meticulous attention to supportive care in terms of temperature regulation, fluid electrolyte balance and nutrition.

Majority of the deaths occurred in the first week $(83.5 \%)$ especially the first 24 hours $(41 \%)$ of admission. The cause being predominantly respiratory related (pneumonia and apnoec attacks). Mortality in the initial hours of admission is usually a reflection of the quality of emergency care(12). The average age at admission of 9.6 days may imply that the parents delayed in bringing their infants for medical attention and only came when the infants were in terminal stages hence the high mortality in the first 24 hours.

As earlier stated, supportive care is an important aspect of care for neonates. The inadequency of supportive care as shown in this study was responsible for a substantial amount of the mortality. Musoke and Revathi researching in the same hospital also found that lack of supportive care for neonates contributed significantly to morbidity and mortality(1). The impact of this poor supportive care is bound to be magnified in a non-specialised area such as the general wards. The most frequent diagnoses in this study differ from those from new born units in the amount of pneumonia, omphalitis, tetanus and hypothermia reported. Adejuyigbe and Musoke also found high incidence of omphalitis in their different studies $(8,12)$. The diagnoses of pneumonia in this study was mainly based on W.H.O clinical criteria(13). Since there are other possible causes of respiratory distress in neonates, and no bacteriological and limited radiological evidence was elicited, there is a chance of over diagnosis. On the other hand, with an average age at admission of 9.6 days, it is possible to have had substantial community acquired pneumonia in these neonates.

Tetanus was mainly a problem of infants born at term and at home with no prior antenatal care. This being within an urban setting, it brings home the reality that not all prospective mothers can access antenatal care. Probable sepsis was the most frequent diagnosis and also the least investigated. This lack of investigation is also reflected in the $29 \%$ of those who had no form of investigation at all. This finding is in agreement with Musoke and Revathi's finding in the new born unit of the same hospital(1). The lack of investigation could have had a background of lack of specimen bottles, night time or weekend because in the year of study, there was no 24 hour, seven days a week laboratory backup (This was enforced two years later). Even when investigations were done, at times the results were never received in the ward or were received too late to be of any meaningful value in the care of the patient. In a situation like that, the clinician may not be keen to investigate. While the number of culture confirmed sepsis are few, it is note worthy that there appears to be an equal distribution between gram positive and gram negative organisms. A finding of predominance of gram positives was reported from Nigeria(12). As in this study, the Nigerian study had substantial part of the study population being born at a place other than the hospital of eventual admission.

The older the baby, the higher the chance of community acquired organisms which will show a tendency towards gram positive bacteria. Most reports from developing countries tend to implicate gram negative bacteria as the common cause of neonatal septicaemia $(8,10,14)$. In this study, there was no case of Lancefield group B-Beta haemolytic streptococci. This is in contrast to the findings of Onyango and coworkers researching in the same wards in 1984 (15) or Moreno et al. in 1987-92 in Latin America (10). The presence of confirmed septicaemia appeared to be associated with prolonged duration of stay in the wards from the average of nine days to 16.6 days. This is a frequent finding by most researchers $(1,10,12)$.

The diagnosis of jaundice was the next most 
frequent. The cause was not established for most. This was also found to be so from previous studies $(2,3)$. This is in contrast to a study from Mulago hospital Kampala where the rate of jaundice was less than $1 \%$ (16). The rate of exchange transfusion (10\%) was in keeping with findings elsewhere. This could be a reflection of inefficient phototherapy.

From this study, there is apparent unjustified use of antibiotics which could lead to increased bacterial multi drug resistance in future. This set back has been noted previously at the same hospital $(1,14)$. The independent predictors of mortality identified in this study only differ in the addition of pneumonia and lack of investigations as predictors. The lack of investigations as a predictor could be spurious in the sense that most deaths occurred within 24 hours of admission probably not giving ample time for relevant investigations to be carried out. Similarly, the diagnosis of pneumonia as previously stated may have been justified though based on clinical criteria.

The policy of the hospital is that for admission in the new born unit, the infant should have been born in $\mathrm{KNH}$ and not yet left hospital, or been referred from another similar unit or if born out of KNH should be 24 hours or less of age. Only in exceptional circumstances are newborns who do not meet the above criteria admitted to the new born unit. An alternative approach would be to set up another neonatal unit for these infants although a study in Larkana-Pakistan suggests that though the number of admissions might increase, the disease patterns and mortality rates may not be altered(17).

In view of this, the benefit of setting up such a unit might only be in being able to concentrate specialized human and material resources at one place and hence reduce costs. Given the findings of this study, the best approach might be to institute selective interventions such as good infection surveillance and control, supportive care such as resuscitation or advanced paediatric life support training for staff. Dawodu and Effiong found that outcome for neonates weighing over $2500 \mathrm{~g}$ was improved when such selective interventions were undertaken in a new born unit without intensive care facilities (18). The majority of deaths occurred in the first week especially the first twenty four hours. This is a reflection of the lack of intensive care facilities for the initial resuscitation and stabilization of these infants. The later deaths are a reflection of the quality of continuing medical care.

Conclusion: The care of neonates in the general paediatric wards is not optimal with excessive mortality through all weight categories especially the low body weight. There is poor supportive care for nutrition, investigations, fluid therapy and temperature control.

\section{RECOMMENDATIONS}

Selective interventions instituted in the wards will help bring down the high mortality rate and avoid having to set up another neonatal unit. This selective interventions may include but not be restricted to;

(i) Training personnel in the wards on paediatric life support (basic and advanced).

(ii) Improved supportive care regarding temperature control, fluid/electrolyte management, nutrition, adequate 24 hours, seven days a week laboratory and other diagnostic services for those who may require the service.

(iii) Improved access to the intensive care unit for those infants who may require the services.

(iv) Improved infection surveillance and control.

\section{ACKNOWLEDGEMENTS}

To the director of Kenyatta National Hospital for permission to publish this paper, Janet Musia for data entry and analysis, Susan Anyango and Foska Osimbo for providing secretarial services.

\section{REFERENCES}

1. Musoke, R. N. and Revathi, G. Emergence of multi drug resistant gram Negative organisms in a neonatal unit and the therapeutic implications. J. Trop. Pediatr. 2000; 46:86-91.

2. Meme, J. S. A prospective study of neonatal deaths in Nairobi, Kenya. East Afr. Med. J. 1978; 55:262-267.

3. Kasirye Bainda E. and Musoke R. N. Neonatal morbidity and mortality at Kenyatta National Hospital-new born unit. East Afr. Med. J. 1992; 69:360-365.

4. Admission and mortality data for the year 2000. Kenyatta National Hospital records department.

5. Usher, R. H. The role of the neonatologist. Pediatr. Clin. North Am. 1970; 17:1999-2008.

6. Dawodu, A. H and Effiong, C.E. Neonatal morbidity and mortality among Nigerian infants in a special care unit. East Afr. Med. J. 1983; 60:39-41.

7. Chintu, C. and Sukhani, S. Perinatal and neonatal morbidity and mortality in Lusaka. Med. J. Zambia. 1978; 12:110.

8. Musoke, R. N. and Malenga G. J. Bacterial infections in neonates at the Kenyatta National Hospital nursery. A retrospective study. East Afr. Med. J. 1984; 61:376-381.

9. Mcintire, D. D. and Bloom, S. L. Birth in relation to morbidity and mortality among newborn infants. N. Engl. J. Med. 1999; 340:1234-1238.

10. Moreno, M. T., Sonia V. et al. Neonatal sepsis and meningitis in a developing Latin American country. Pediatr. Infect. Dis. J. 1994; 13:516-520.

11. Were, F. N., Mukhwana, B. O, and Musoke, R. N. Neonatal survival of infants less than 2000 grams born at Kenyatta National Hospital. East Afr. Med. J. 2002; 79:77-79.

12. Adejuyigbe, E. A, Adeodu, O.O., Ako-Nai K.A et al. Septicaemia in high risk neonates at a teaching hospital in Ile-Ife, Nigeria. East Afr. Med. J. 2001; 70:540-543.

13. A programme for controlling acute respiratory infection in children. Memorandum from WHO meeting. Bull. Wld. Hlth. Org. 1984; 62:47-58.

14. Musoke, R. N. Rational use of antibiotics in neonatal infections. East Afr. Med. J. 1997; 74:147-150.

15. Onyango, F.E, Ndinya-Achola J. Musoke R.N. et al. Lancefield group B Beta-haemolytic streptococcol infections in the newborn at Kenyatta National Hospital. East Afr. Med. J. 1984; 61:376-381.

16. Musoke R. N. Admissions to Mulago hospital special care baby unit. 1971-1975, a retrospective study. East Afr. Med. J. 1983; 60:699-703.

17. Abbasi K. A. Neonatal disease profile in Larkana before and after establishment of a neonatal ward. J. Pak. Med. Assoc. 1995; 45:235-236.

18. Dawodu A. H. and Effiong C. E. Neonatal mortality: effects of selective pediatric interventions. Paediatrics. 1985; 75: 51-57. 